\title{
Incautious Use of Antibiotics During Covid-19
}

\author{
Faiza Zeeshan
}

\begin{abstract}
How to cite this Article:
Zesshan F. Incautious Use of Antibiotics During Covid-19. J Bahria Uni Med Dental Coll. 2022; 12(1):60-62 DOI: https://doi.org/ 10.51985/JBUMDC2021090

This is an Open Access article distributed under the terms of the Creative Commons Attribution Non Commercial License (http:// creativecommons/org/licences/by-nc/4.0)

which permits unrestricted non commercial use, distribution and reproduction in any medium, provided the original work is properly cited.
\end{abstract}

The severe acute respiratory syndrome corona virus (SARSCoV-2), the etiologic agent of the most detrimental disease of the century, has tragically influenced the world dynamics. One of the major challenges faced by health sector globally, was to establish a treatment regimen and guidelines to combat this lethal condition. The World Health Organization (WHO) advocates early empiric antibacterial medication with continuous scrutiny for downscaling of signs and symptoms in the patients with severe COVID-19. ${ }^{1}$ Nevertheless, serious queries have driven in the context of use of irrational antibiotics in the time where the world is already on the verge of antimicrobial resistance. In 2019, the UN Interagency Coordination Group on Antimicrobial Resistance alarmed that by 2050, antibiotic-resistant diseases would be the culprit for 10 million deaths each year and can be calamitous by 2030 , and antimicrobial resistance could put 24 million people into extreme scarcity of resources. ${ }^{2}$ The antibacterial resistance is another pandemic which is silently approaching the millennials and netizens, irrespective of their oblivion.

The most commonly prescribed antibiotics during COVID19 pandemic are found to be fluoroquinolones, macrolides and cephalosporins. ${ }^{3}$ The basic purpose of the antimicrobials drugs is to curb and to prevent secondary bacterial infections in the COVID-19 pneumonia patients. However, there is a paucity of supporting data regarding the association of coronavirus related respiratory illness and superimposed bacterial infections. Around 8\% cases of COVID-19 globally presented with bacterial and fungal co infections so far. ${ }^{4}$ Study from UK demonstrated 3.2\% SARS-CoV-2 patients developed secondary bacterial infections. ${ }^{5} \mathrm{Li}$ and co researchers from Wuhan, China showed 6.8\% COVID-19 patients developed hospital associated infections. ${ }^{6}$ The commonest observed offenders are Acinetobacter baumanaii, Klebsiella pneumoniae, Methicillin resistant Staphylocooccus aurues, Pseudomonas, Candida, Mycoplasma, and Cryptococcus. Despite of lower rates of bacterial co infect-

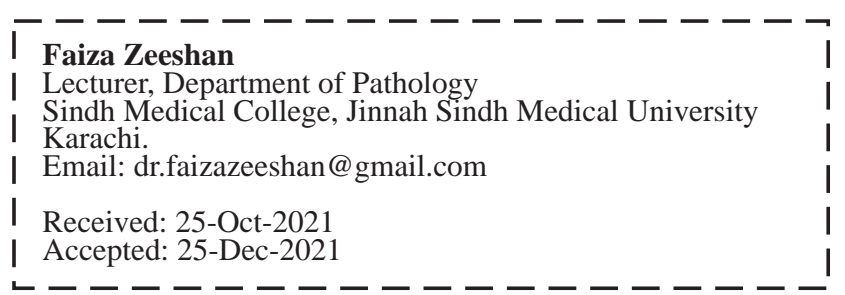

ions, surprisingly higher rates of antibiotic prescriptions are reported. A study from China revealed around $99 \%$ critical and non-critical patients received antibacterial therapy including cephalosporin, carbapenems and quinolones, although none of the cases were found to be co infected with bacterial or fungal pathogens. ${ }^{4}$ Similarly, a study by Guan et al reported 58\% out of 1099 patients were given intravenous antibiotics. ${ }^{7}$ A review analysis by Rawson and his colleagues showed that antibiotics were given in $72 \%$ cases of COVID-19 however, only $8 \%$ were found to be co infected with bacterial species. ${ }^{3}$ The preliminary results by Buetti et al illustrated that early administered antibiotics had no impact on significant reduction of mortality or delayed hospital-acquired infections in critically ill patients. ${ }^{8}$

The role of antibiotics in the treatment of COVID-19 is still debatable. The use of hyrdoxychloroquine alone or in combination with azithromycin has been recommended by clinicians worldwide for the treatment of SARS-CoV-2 patients. Although the polarity has been observed regarding the effectiveness of these drugs in terms of reduced morbidity, span of hospitalization and case fatalities especially in elderly patients with ischemic heart diseases. The drug-drug interaction has found to be responsible for prolonged QT intervals and torsades de pointes along with the potential threat of antibiotic resistance. ${ }^{9}$

One of the possible reasons for commencement of empirical antibiotic therapy for SARS-CoV-2 patients is synergy which exists between viral and bacterial infections, as it has been observed with influenza viruses. However, strong evidence is still required. It has been observed that respiratory viruses are responsible for immune paralysis, a condition in which antigen presenting cells (macrophages and dentritic cells) are overwhelmed by the load of apoptotic lung cells owing to viral infection. Consequently, there is an increase in bacterial pathogens growth. The viral infections disrupt mucocilliary apparatus and cause thickening of the mucus resulting impaired movement of immune cells. If such stipulated synergy is proven, the usage of antibiotics would be fruitful, otherwise, it is cumbersome. ${ }^{10}$ Currently the beneficial effects of antibiotics in context of SARS-CoV-2 is still debatable, however, the evidences of impending side effects exist.

Several reports have come in lime light depicting the increased rate of antibiotic resistance in the dark era of covid 
pandemic. The Carbapenem Resistant Enterobacteriaceae (CRE) have been reported in French COVID-19 positive population. This can be associated with irrational use of antibiotics. Some of the studies reported exaggerated symptoms of COVID-19 illness in the patients of gastrointestinal (GIT) problems. Provided that antibiotics can disturb the gut microbiome, hence further complicating the GIT issues and emergence of resistant bacterial strains. Therefore, empirical antibiotics should be prescribed cautiously. ${ }^{11}$ Another noteworthy finding is association of frequent sanitizers and disinfectants to disarray of natural environmental niche of microbiota, causing emergence of various resistant superbugs, for instance alcohol resistant strain of Enterococcus faecium has recently been reported in Australia. ${ }^{12}$ Undriven antibiotic steward programs, curtailing of hospitalization for bacterial infections, limited diagnostic abilities due to telemedicine are some of the factors which have promoted the unnecessary prescription of antibiotics and eventually rise in antibiotic resistance.

The crème de la crème approach to prescribe antibiotics for viral illness generally and SARS-CoV-2 specifically, based on evidences, is to seek for COVID-19 most and least common compatible symptoms. The commonest symptoms are fever, dry cough and lethargy, while the uncommon symptoms include sore throat, diarrhea, conjunctivitis, headache, loss of taste or smell, skin rashes and discoloration. ${ }^{13}$ Antibiotics should be prescribed to the patients experiencing the worst signs and symptoms for example those with low oxygen saturations and rapidly developing respiratory failure. Biomarkers including Creactive protein, procalcitonin, and serum ferritin may play a role in deciding for patient selection, but this needs further evaluation. The thumb rule to follow is "Patients without severe respiratory complaints should be managed without antibiotics". In hospital settings, the diagnostic work-up includes a thorax CT scan, this offers more findings of the typical infiltrates related with bacterial lower respiratory tract infections as contrary to the typical glass ground opacities observed in COVID-19. This extra diagnostic approach should permit physicians to hold back the empirical antibiotics in patients with peculiar images for COVID-19 on CT scan. ${ }^{14}$ Antibiotic regimen should be properly followed as per the standard protocol. The important biomarkers should be considered for stopping the antibiotics, once the treatment plan is completed. A beta lactam coverage is required for $S$. pneumoniae and Methicillin resistant $S$. aureus (MRSA) preferably once a day administration. For atypical coverage, preference should be given to doxycylcline. If secondary respiratory worsening is suspected, readministration of antibiotics should be made only when there is a bacterial culture evidence, as in most cases the deterioration in symptoms is associated with hyper inflammatory responses rather than secondary bacterial infections. ${ }^{14}$
The use of proper personal protective equipment has proven to be the main source of dissipating bacterial infections during COVID-19 pandemic. Hand hygiene measures are mandatory and all health workers should adopt World Health Organization (WHO) 5 moments for hand hygiene approach. ${ }^{15}$ This can help in curbing spread of nosocomial infections. Antibiotic stewardship programs (ASP) bears a pivotal role in traditional health system. ASPs can evolve strategies to spot the patients with COVID-19-like-illness; this is specifically fruitful when these cases are overlooked initially. ASPs can also aid in the management of potential drug shortages, establishing local treatment guidelines, optimizing the use of antibiotics, and in the diagnostic stewardship of COVID-19 testing. Unfortunately in Pakistan, the role of ASP is still in the budding phase, despite the fact, irrational use of antibiotics is a norm in our clinical practice. The usage of antibiotics was higher during the COVID-19 pandemic as compared to the pre-pandemic period: the consumption of azithromycin raised from 11.5 daily define doses (DDD) per 100 occupied bed-days in 2019 to 17.0 DDDs per 100 occupied bed-days in 2020, while the administration of ceftriaxone escalated from 20.2 DDDs per 100 occupied bed-days in 2019 to 25.1 DDDs per 100 occupied bed-days in $2020 .{ }^{16}$ This can be alarming and warrants strict health interventions and their implementations by the stake holders.

Prompt diagnosis of COVID-19 is multifactorial, which can be achieved by developing communication strategies with patients, COVID-19 testing, and symptoms management plans. They all can contribute to damper injudicious use of antibiotics and saving mankind from an upcoming challenge.

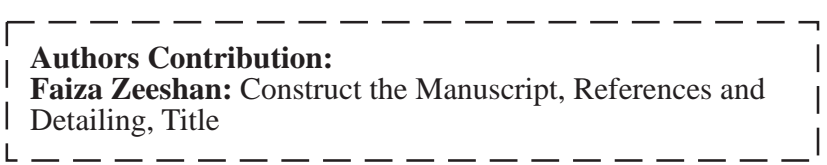

\section{REFERENCES:}

1. Zhu N, Zhang D, Wang W, et al. A novel coronavirus from patients with pneumonia in China, 2019. N Engl J Med 2020; 382:727-33. https:// doi.org/ 10.1056/NEJMoa2001017.

2. Ginsburg AS, Klugman KP. COVID-19 pneumonia and the appropriate use of antibiotics. The Lancet Global Health. 2020;8(12):e1453-4. https://doi.org/10.1016/S2214-109X (20) 30444-7.

3. Chedid, M., Waked, R., Haddad, E., Chetata, N., Saliba, G., \& Choucair, J. (2021). Antibiotics in treatment of COVID19 complications: a review of frequency, indications, and efficacy. Journal of infection and public health, 14(5), 570-576.

4. Rawson TM, Moore LS, Zhu N, Ranganathan N, Skolimowska K, Gilchrist M, Satta G, Cooke G, Holmes A. Bacterial and fungal coinfection in individuals with coronavirus: a rapid review to support COVID-19 antimicrobial prescribing. Clinical Infectious Diseases. 2020;71(9):2459-68. https:// doi.org/10.1093/cid/ciaa530. 
5. Sharifipour E., Shams S., Esmkhani M., Khodadadi J., FotouhiArdakani R., Koohpaei A. Evaluation of bacterial co-infections of the respiratory tract in COVID-19 patients admitted to ICU. BMC Infect Dis. 2020;20:646. https://doi.org/ 10.1186 /s12879-020-05374-Z.

6. Li J., Wang J., Yang Y., Cai P., Cao J., Cai X. Etiology and antimicrobial resistance of secondary bacterial infections in patients hospitalized with COVID-19 in Wuhan, China: a retrospective analysis. Antimicrob Resist Infect Control. 2020;9:153. https://doi.org/10.1186/s13756-020-00819-1.

7. Guan W.J., Ni Z.Y., Hu Y., Liang W.H., Ou C.Q., He J.X. Clinical characteristics of coronavirus disease 2019 in China. N Engl J Med. 2020;382:1708-1720. https: //doi.org/ 10.1056 /NEJMoa2002032.

8. Buetti N, Mazzuchelli T, Priore EL, Balmelli C, Llamas M, Pallanza M, Elzi L, Consonni V, Trimboli P, Forni-Ogna V, Bernasconi E. Early administered antibiotics do not impact mortality in critically ill patients with COVID-19. Journal of Infection. 2020;81(2):e148-9.https://doi.org/ 10.1016/j.jinf .2020.06.004

9. Jankelson L, Karam G, Becker ML, Chinitz LA, Tsai MC. QT prolongation, torsades de pointes, and sudden death with short courses of chloroquine or hydroxychloroquine as used in COVID-19: A systematic review. Heart rhythm. 2020;17(9):1472-9. https://doi.org/ 10.1016/j.hrthm. 2020. 05.008 .

10. Manohar Prasanth, Loh Belinda, Athira Sudarsanan, Nachimuthu Ramesh, HuaXiaoting, Welburn Susan C, et al. Secondary Bacterial Infections DuringPulmonary Viral Disease: Phage Therapeutics as Alternatives to Antibiotics?.Front Microbiol 2020;11:1434. https://doi.org/ 10.3389/fmicb.2020.01434
11. Kim H.S. Do an altered gut microbiota and an associated leaky gut affect COVID-19 severity? mBio. 2021;12(1) e03022-20. https://doi.org/10.1128/mBio.03022-20.

12. Singh A. COVID-19: Disinfectants and sanitisers are changing microbiomes. Bmj. 2020;370. DOI: https://doi.org/ 10.1136/bmj.m2795.

13. Leis JA, Born KB, Theriault G, Ostrow O, Grill A, Johnston $\mathrm{KB}$. Using antibiotics wisely for respiratory tract infection in the era of COVID-19. bmj. 2020;371. https://doi.org/ 10.1136/bmj.m4125.

14. Claessens Y.E, Debray M.P, Tubach F, Brun A.L, Rammaert B, Hausfater P. Early chest computed tomography scan to assist diagnosis and guide treatment decision for suspected community-acquired pneumonia. Am J Respir Crit Care Med. 2015;192:974-982. https://doi.org/10.1164/rccm.2015010017OC

15. Van Nguyen H, Tran HT, Khuong LQ, Van Nguyen T, Ho NT, Dao AT et al. Healthcare Workers' Knowledge and Attitudes Regarding the World Health Organization's "My 5 Moments for Hand Hygiene": Evidence From a Vietnamese Central General Hospital. Journal of Preventive Medicine and Public Health. 2020;53(4):236. https://doi.org/ 10.3961/jpmph.19.319.

16. Mustafa ZU, Salman M, Aldeyab M, Kow CS, Hasan SS. Antimicrobial consumption among hospitalized patients with COVID-19 in Pakistan. SN comprehensive clinical medicine. 2021:1-5. https://doi.org/10.1007/s42399-021-00966-5. 